\title{
Soluble Transferrin Receptor and Soluble Transferrin Receptor/Log Ferritin Ratio are Correlated with Iron Status in Regular Hemodialysis Patients
}

\author{
Yenny Kandarini ${ }^{1, *}$, Gede Wira Mahadita ${ }^{1}$, Sianny Herawati ${ }^{2}$, Anak Agung Wiradewi Lestari², \\ Ketut Suega ${ }^{3}$, I Gde Raka Widiana ${ }^{1}$ \\ ${ }^{1}$ Nephrology and Hypertension Division, Internal Medicine Department, Faculty of Medicine, Universitas Udayana/Sanglah General Hospital, \\ Jl. P.B. Sudirman, Denpasar, Bali, Indonesia \\ ${ }^{2}$ Clinical Pathology Department, Faculty of Medicine, Universitas Udayana/Sanglah General Hospital, J1. P.B. Sudirman, Denpasar, Bali, Indonesia \\ ${ }^{3}$ Hematology Oncology Division, Internal Medicine Department, Faculty of Medicine, Universitas Udayana/Sanglah General Hospital, \\ Jl. P.B. Sudirman, Denpasar, Bali, Indonesia \\ *Corresponding author. E-mail: yenny_kandarini@unud.ac.id
}

Received date: Sep 29, 2020; Revised date: Apr 29, 2021; Accepted date: May 4, 2021

\section{Abstract}

$\mathrm{B}$ ACKGROUND: Monitoring of iron status in chronic kidney disease patients is important, however inflammation may hinder its monitoring. Soluble transferrin receptor (sTfR) is an alternative parameter to overcome this issue, whereas ferritin play a part in the inflammation process. Hence, the correlation between the sTfR ratio and the sTfR/log ferritin ratio with conventional iron status parameters in regular hemodialysis patients is necessary to be evaluated.

METHODS: A cross-sectional was conducted in the current study. As many as $5 \mathrm{~mL}$ of blood $(2 \mathrm{~mL}$ for sTfR and $3 \mathrm{~mL}$ for serum iron and ferritin levels) was collected. sTfR level was the blood-soluble transferrin receptor level measured by the enzyme-linked immunosorbent assay (ELISA). The amount of ferritin and serum iron was determined using the immunochemiluminescent process. To evaluate the correlation, the Pearson correlation test was used.
RESULTS: A total of 80 subjects was included in this study. The mean of hemoglobin was $10.25 \pm 1.66 \mathrm{~g} / \mathrm{dL}$, serum iron was $58.19 \pm 26.56 \mathrm{~g} / \mathrm{dL}$, and the median ferritin was 520.4 (49.9-3606) $\mathrm{ng} / \mathrm{mL}$. The sTfR was significantly associated only with serum iron levels with a correlation coefficient of $\mathrm{r}=-0.242 ; p=0.031$. The $\mathrm{sTfR} / \log$ ferritin was significantly associated with serum iron 1 evels (InSI) $(\mathrm{r}=-0.255, p=0.022)$; and transferrin saturation $(\mathrm{r}=-0.295$; $p=0.008$ ).

CONCLUSION: sTfR/log ferritin has a negative and significant correlation with serum iron levels and transferrin saturation, while sTfR negatively correlated with serum iron levels. sTfR and sTfR/log ferritin may be considered as an alternative iron marker in inflammation setting such as $\mathrm{CKD}$.

KEYWORDS: sTfR/log ferritin, iron status, serum iron, ferritin, chronic kidney disease, hemodialysis

Indones Biomed J. 2021; 13(2): 201-7

\section{Introduction}

Chronic kidney disease (CKD) is one of the most common diseases in populations worldwide, with a high incidence and prevalence and requiring enormous treatment costs. Anemia is a condition that is often found in chronic kidney disease, which can develop at an early stage of the disease and decrease the quality of life. The prevalence of CKD worldwide is estimated at $10 \%$. More than $80 \%$ of endstage CKD patients who receive renal replacement therapy are spread across developing countries.(1)

Various factors can cause anemia in chronic kidney disease. Erythropoiesis and iron homeostasis can be impaired due to elaborate events, including relative erythropoietin deficiency, chronic inflammation, loss of blood, reduced 
absorption and use of iron, and non-regulated biological processes due to the administration of exogenous iron, erythropoietin, and blood transfusions.(2)

The administration of erythropoiesis-stimulating agents and various intravenous iron preparations gives more effective results in managing anemia in chronic kidney disease, maintains hemoglobin levels in a specific range, and effectively treats iron deficiency.(2)

Monitoring of iron status in patients with chronic kidney disease who have received erythropoietin treatment is crucial for detecting iron deficiency and preventing side effects due to iron therapy. Assessment of iron requirements and monitoring of iron therapy requires accurate markers of iron status. The commonly used iron status assessment is examining serum ferritin, serum iron, and transferrin saturation. However, in some conditions, these laboratory markers do not offer enough clinical information, so that a better alternative markers are needed, one of which is the soluble transferrin receptor (sTfR). $(2,3)$

The sTfR is a protein composed of polypeptide chain formed from the transferrin receptor. This cellular protein is expressed primarily in iron-requiring cells. Plasma sTfR concentrations reflect the receptor density on cells and the number of cells that express the receptors, so they are near related to the need for iron in cells and the rate of erythroid proliferation. $(2,3)$

Ferritin and transferrin concentrations may be used as monitoring of iron status, However, they may influenced by chronic diseases or inflammation. In contrast to ferritin and transferrin concentrations in serum, sTfR is not influenced by chronic disease conditions or inflammation. Distinguishing the chronic disease anemia and iron deficiency anemia is important, as well in situations of combined iron deficiency anemia and chronic disease anemia. However, to be able to differentiate those pathologies, novel parameters is mandatory. The sTfR concentration will increase in iron deficiency anemia, but not in chronic disease anemia, hence it may be able to differentiate the two conditions. $(2,3)$ In addition, compared to sTfR measurement only, the use of the sTfR/log ferritin ratio has advantages in conditions influenced by these two parameters on iron deficiency anemia, such as increased sTfR and decreased ferritin. It may increase the diagnostic efficacy. $(2,3,4)$

The study which assesses sTfR and sTfR/log ferritin with conventional iron parameters, particularly in hemodialysis patients receiving erythropoietin maintenance, has never been done. Based on this background, this study was conducted to determine the correlation of sTfR and $\mathrm{sTfR} / \log$ ferritin ratio with the iron status parameters commonly used, namely serum iron, transferrin saturation, and ferritin in chronic kidney disease patients undergoing hemodialysis.

\section{Methods}

\section{Sample Recruitment}

This was an analytical observational study with a crosssectional approach to determine the correlation between the $\mathrm{sTfR} / \log$ ferritin ratio and the iron status (serum iron and ferritin) in patients with chronic kidney disease undergoing hemodialysis. The target population of the study was chronic kidney disease patients undergoing hemodialysis at Sanglah General Hospital, Denpasar. The research sample was chronic kidney disease patients undergoing hemodialysis at Sanglah General Hospital, patients aged more than 18 years, not in pregnancy, and willing to participate were included in this study. The sample size was determined using the formula, which resulted in 73 samples in total.

This study was approved by the ethical board of faculty of medicine of Udayana University/Sanglah Hospital with the number of $1872 / \mathrm{UN} 14.2 .2$.VII.14/LP/2018. The research was conducted in all inpatient rooms of Sanglah Hospital Denpasar and Clinical Pathology Laboratory of Sanglah Hospital Denpasar. The time for collecting the research sample was carried out from April 2018 until the number of samples was met. The sTfR/log ferritin ratio was the ratio of sTfR levels examined by the enzyme-linked immunosorbent assay (ELISA) method with the logarithm of ferritin levels. Serum iron levels were measured by the colorimetric method.

\section{Measurement of sTfR, Ferritin and Serum Iron}

As many as $5 \mathrm{~mL}$ of blood $(2 \mathrm{~mL}$ for sTfR and $3 \mathrm{~mL}$ for serum iron and ferritin levels) of patients with chronic kidney disease who undergo hemodialysis and meet the criteria for acceptance of the sample were collected. Blood collection for this examination was carried out in conjunction with blood collection for other routine laboratory examinations).

Examination of sTfR, ferritin, and serum iron was carried out on blood samples. The tube centrifuged at 3,000 rpm for 15 minutes, then made plasma aliquots and immediately stored at $-80^{\circ} \mathrm{C}$. The sTfR examination was carried out after the total number of samples was met using the ELISA method. The reagent for sTfR examination used was Quantikine ${ }^{\circledR}$ ELISA Human sTFR (Cat: DTFR1, R\&D Systems, Minneapolis, USA). The measurement was performed with Microplate Reader 
Biorad model 680 (Bio-rad Laboratories, Hercules, CA, USA) with Microplate Manager Software ver 5.2.1 (Biorad Laboratories). Meanwhile, ferritin and serum iron examination was immediately carried out using the electrochemiluminescence immunoassay (ECLIA) method. The $\mathrm{sTfR} / \log$ ferritin ratio was obtained by performing manual calculations/calculations of sTfR levels and ferritin levels.

\section{Statistical Analysis}

Statistical analysis was done with SPSS version 23 software (IBM Corporation, Armonk, NY, USA). Descriptive statistics were employed to describe the characteristics of research subjects. A correlation test was done to see the correlation between sTfR and sTfR/log ferritin ratio with serum iron and ferritin with the Pearson correlation test. The statistical analysis used a $p$-value $<0.05$.

\section{Results}

\section{Characteristics of the Study}

A total of 80 patients, consisting of 52 men and 28 women, were involved in this study. Ten samples (12.5\%) had iron deficiency anemia. Each of 5 people $(6.25 \%)$ had absolute iron deficiency anemia and functional iron deficiency anemia. The mean age was $52.06 \pm 13.21$ years. The mean for sTfR/log ferritin was $1.07 \pm 0.43$. Details of the demographic characteristics, anthropometric characteristics, laboratory characteristics, and iron status were presented in Table 1.

Several variables in this study had abnormal data distribution after the data normality test was carried out with the Kolmogorov-Smirnov test, i.e., the levels of high-sensitivity c reactive protein (HsCRP) $(p=0.000)$, SI $(p=0.02)$, ferritin $(p=0.003)$, and hepcidin $(\mathrm{p}=0.002)$. A logarithmic transformation was performed for variables with abnormal data distribution to qualify for the parametric test with Pearson correlation. After the logarithmic transformation was carried out and tested again with the Kolmogorov-Smirnov test, the normal distribution for the transformed logarithmic variable (In) CRP (InHsCRP) ( $p=0.568), \log$ transformation (In)SI $(p=0.623)$, and InHepcidin $(p=0.445)$ was obtained.

\section{Correlation of $s$ TfR, sTfR Index with Iron Parameters}

The sTfR was significantly associated with serum iron levels. From the Pearson correlation analysis between sTfR and InSI, the correlation coefficient $\mathrm{r}=-0.242 ; p=0.031$. The correlation obtained was negative, indicating that the higher the sTfR level, the lower the serum iron level.
Table 1. Characteristics of subjects.

\begin{tabular}{|c|c|}
\hline $\begin{array}{l}\text { Characteristic } \\
\qquad(\mathrm{n}=\mathbf{8 0})\end{array}$ & Value \\
\hline \multicolumn{2}{|l|}{ Gender } \\
\hline Male, n (\%) & $52(65 \%)$ \\
\hline Female, n $(\%)$ & $28(35 \%)$ \\
\hline Age (years), mean $\pm \mathrm{SD}$ & $52.06 \pm 13.21$ \\
\hline BMI $\left(\mathrm{kg} / \mathrm{m}^{2}\right)$, mean $\pm \mathrm{SD}$ & $21.62 \pm 4.06$ \\
\hline Hemoglobin (gr/dL), mean \pm SD & $10.25 \pm 1.66$ \\
\hline $\operatorname{MCV}(\mathrm{fl})$, mean $\pm \mathrm{SD}$ & $90.28 \pm 6.52$ \\
\hline $\mathrm{MCHC}(\%)$, mean $\pm \mathrm{SD}$ & $32.41 \pm 0.95$ \\
\hline $\mathrm{MCH}(\mathrm{pg})$, mean $\pm \mathrm{SD}$ & $29.28 \pm 2.45$ \\
\hline $\mathrm{RDW}(\%)$, mean $\pm \mathrm{SD}$ & $14.39 \pm 1.42$ \\
\hline $\mathrm{SI}(\mu \mathrm{g} / \mathrm{dL})$, mean $\pm \mathrm{SD}$ & $58.19 \pm 26.56$ \\
\hline $\operatorname{TIBC}(\mu \mathrm{g} / \mathrm{dL})$, mean $\pm \mathrm{SD}$ & $203.61 \pm 36.89$ \\
\hline Ferritin (ng/mL), median (min-max) & $520.4(49.9-3606)$ \\
\hline Transferrin saturation $(\%)$, mean $\pm \mathrm{SD}$ & $28.64 \pm 10.90$ \\
\hline HsCRP (mg/L), median (min-max) & $5(0.4-192.9)$ \\
\hline $\mathrm{HsCRP} \leq 10, \mathrm{n}(\%)$ & $54(67.5 \%)$ \\
\hline HsCRP $>10, \mathrm{n}(\%)$ & $26(32.5 \%)$ \\
\hline $\mathrm{ESR}$ (mm/hours), mean $\pm \mathrm{SD}$ & $46.6 \pm 25.34$ \\
\hline $\operatorname{sTfR}(\mu \mathrm{g} / \mathrm{mL})$, mean $\pm \mathrm{SD}$ & $2.86 \pm 1.02$ \\
\hline $\mathrm{sTfR} / \log$ Ferritin, mean $\pm \mathrm{SD}$ & $1.07 \pm 0.43$ \\
\hline Hepcidin (ng/ml), median (min-max) & $6.84(0.40-55.30)$ \\
\hline $\mathrm{BUN}(\mathrm{mg} / \mathrm{dl})$, mean $\pm \mathrm{SD}$ & $66.77 \pm 17.29$ \\
\hline $\mathrm{SC}(\mathrm{mg} / \mathrm{dl})$, mean $\pm \mathrm{SD}$ & $13.53 \pm 4.45$ \\
\hline URR (\%), mean \pm SD & $73.80 \pm 8.77$ \\
\hline Functional IDA, n (\%) & $5(6.25 \%)$ \\
\hline Absolute IDA, n (\%) & $5(6.25 \%)$ \\
\hline
\end{tabular}

BMI: body mass index; MCV: mean corpuscular volume; MCHC: mean corpuscular hemoglobin concentration; $\mathrm{MCH}$ : mean corpuscular hemoglobin; RDW: red cell distribution width; SI: serum iron; TIBC: total iron binding capacity; HsCRP: high sensitivity $\mathrm{C}$ reactive protein; LED: erythrocyte sedimentation rate; sTfR: soluble transferrin receptor; BUN: blood urea nitrogen; SC: Serum creatinine; URR: urea reduction rate; IDA: iron deficiency anemia.

To assess the correlation between the sTfR index (sTfR/log ferritin) and serum iron levels (InSI), Pearson correlation analysis was performed, and a significant negative correlation was obtained with a value of $\mathrm{r}=-0.255$, $p=0.022$. The higher the $\mathrm{sTfR} / \log$ ferritin value, the lower the SI will be. In relation to other iron status parameters, sTfR/ log ferritin was also significantly associated with transferrin saturation (TSat) with a value of $\mathrm{r}=-0.295 ; p=0.008$ (Figure 1 , Table 2). On the other hand, InSI or serum iron was found 

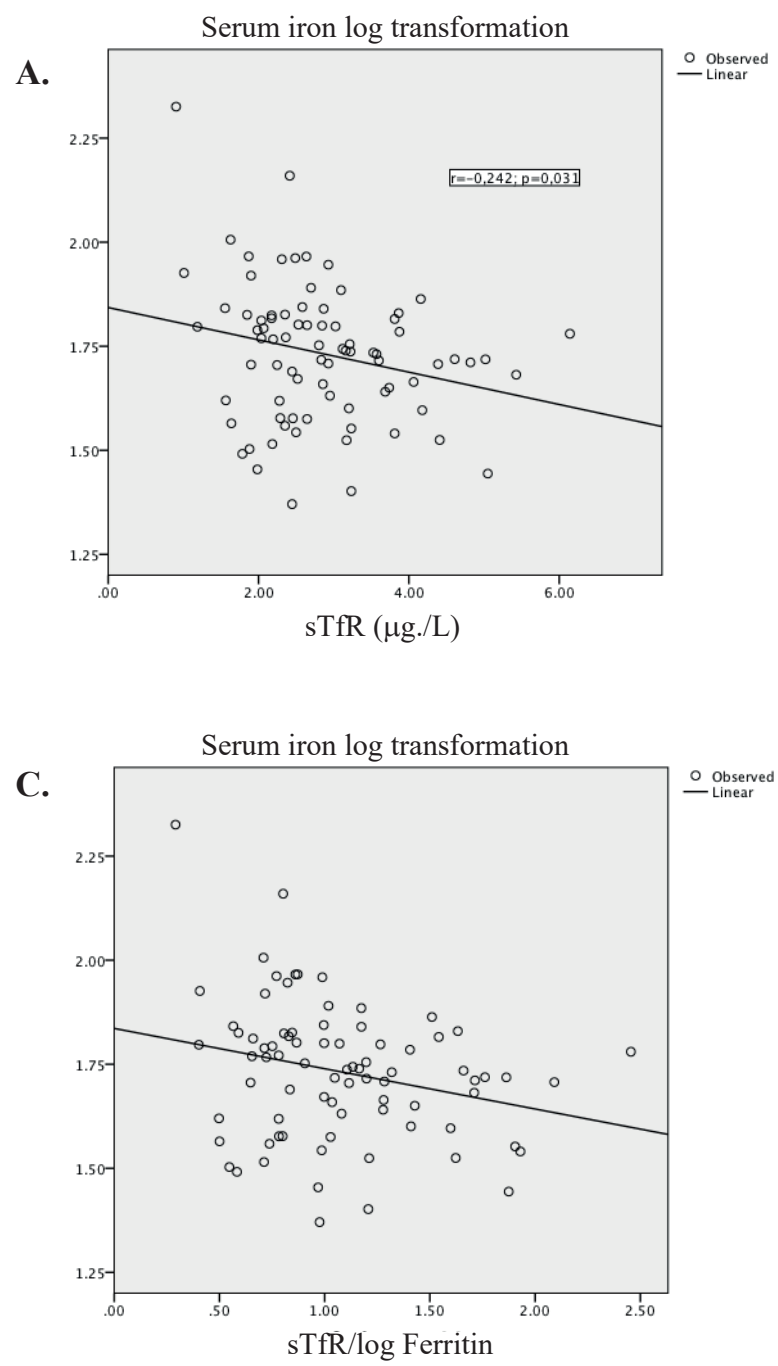

to have no significant correlation with Inferritin, considered iron reserves, with $\mathrm{r}=0.088 ; p=0.438$. Other correlations between sTfR and sTfR/log ferritin with other conventional iron status parameters are depicted in Table 2. The facts from this empirical data can be assumed to occur because of the role of inflammatory factors, as assessed by HsCRP in people with regular hemodialysis.

\section{Correlation with Inflammatory Status}

In this study, there was a significant correlation between inflammatory conditions measured by InHsCRP and serum Inferritin levels with a correlation coefficient of $\mathrm{r}=0.348$;

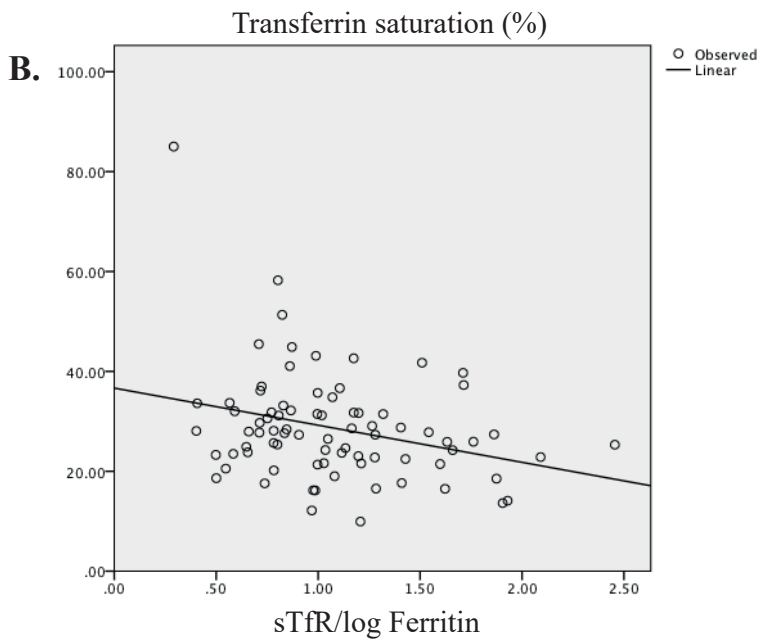

Figure 1. Correlation graph of sTfR, sTfR/log ferritin, InSI, and Tsat. A: sTfR and InSI; B: sTfR/log ferritin and Tsat; C): sTfR/log ferritin and InSI.

$p=0.002$. InHsCRP levels were also significantly associated with InSI, but with a negative correlation, $\mathrm{r}=-0.261$; $p=0.019$. This significant correlation provides an initial picture that several iron status parameters, such as SI and ferritin, are influenced by inflammatory variables in patients undergoing regular hemodialysis. On the other hand, other iron status parameters such as transferrin saturation were not associated with the InHsCRP inflammatory marker $(\mathrm{r}=-0.085 ; p=0.454)$. The significant correlation between InHsCRP, which is considered a marker of inflammation in hemodialysis patients with several iron status variables, is presented in Table 3.

Tabel 2. Correlation of sTfR and sTfR/log ferritin with conventional iron status parameters in regular hemodialysis subjects.

\begin{tabular}{lccc}
\hline & InSI & TSat & InFerritin \\
\hline sTfR & $\mathrm{r}=-0.242 ; p=0.031 *$ & $\mathrm{r}=-0.213 ; p=0.058$ & $\mathrm{r}=-0.195 ; p=0.084$ \\
$\mathrm{sTfR} / \log$ Ferritin & $\mathrm{r}=-0.255 ; p=0.022^{*}$ & $\mathrm{r}=-0.295 ; p=0.008^{*}$ & \\
\hline
\end{tabular}


Tabel 3. Correlation of inflammation parameter of InHsCRP with conventional iron status parameters.

\begin{tabular}{lcc}
\hline & \multicolumn{2}{c}{ InHsCRP } \\
\cline { 2 - 3 } & r & $\boldsymbol{p}$ \\
\hline InSI & -0.261 & $0.019^{*}$ \\
Tsat & -0.085 & 0.454 \\
InFerritin & 0.348 & $0.002^{*}$ \\
sTfR & -0.053 & 0.640 \\
sTfR/log Ferritin & -0.122 & 0.282 \\
Hepcidin & -0.076 & 0.502 \\
\hline
\end{tabular}

\section{Discussion}

The sTfR in this study had a significant correlation only with serum iron levels as iron status. On the other hand, the correlation between sTfR and Tsat and ferritin was not statistically significant. The correlation between sTFR and Tsat or ferritin was not statistically significant.

A previous study involving 49 regular HD patients who routinely received intravenous Epo and iron therapy found that sTfR was also correlated with transferrin saturation ( $\mathrm{r}=$ $0.38 ; \mathrm{p}<0.05)$ but not significantly associated with ferritin. (5) In further studies, sTfR can even be used as a predictor of intravenous iron therapy response in regular HD patients with a cut-off point of about $1.5 \mathrm{mg} / \mathrm{L}$ and superior to ferritin and transferrin saturation.(6) From several explanations of the results of other studies, it was found that the variability of the correlation between sTfR and several other markers of iron status was found. Another study found a significant correlation between sTfR and SI, TSat, and hemoglobin.(7) sTfR negatively correlated with SI, transferrin saturation, and hemoglobin. As for this study, sTfR correlated significantly with serum iron, while sTfR index correlated significantly with serum iron and transferrin saturation.

A prospective multicenter study to differentiate iron deficiency anemia and anemia in chronic disease found that sTfR levels were significantly higher in iron deficiency anemia. ( $p=0.0001)$.(8) Studies comparing the hematological sample set of HD patients with healthy people as controls found that the HD population had higher CRP levels and higher sTfR. Very likely with iron deficiency anemia.(9)

This sTfR marker, in principle, checks for transferrin receptors in serum released from the cell surface of erythroblasts. When free iron in cells decreases, it will be followed by the expression of transferrin receptors on the erythroblast cell surface. sTfR is not related and influenced by inflammatory conditions because it does not include acute phase reactants such as Tsat and ferritin.(10) However, what remains a question and requires further investigation is whether the increase in sTfR that is often obtained after the administration of Epo therapy is a marker of iron deficiency or a response to Epo therapy.(11) The current study found a significant correlation between STfR and serum iron levels.

The results of this study obtained a statistically significant correlation or negative correlation between $\mathrm{sTfR} / \log$ ferritin and serum iron levels. The higher the $\mathrm{sTfR} / \log$ ferritin value, the lower the SI level or vice versa. The significant correlation between sTfR/log ferritin and SI obtained in this study proves that sTfR/log ferritin correlates with one of the iron status parameters. On the other hand, there was also a significant negative correlation between $\mathrm{sTfR} / \log$ ferritin and transferrin saturation. In our analysis, no correlation analysis was carried out between sTfR/log ferritin and serum ferritin levels because one variable is already part of the other variable.

The results obtained in this study are consistent with another previous study who obtained a negative and significant correlation between serum iron levels and the sTfR index (sTfR/log ferritin).(7) sTfR/log ferritin was also found to have a significant correlation with Tsat. The sTfR/ log ferritin was higher in samples with iron-depleted state anemia (bone marrow iron staining score 0 ) and significantly different from samples without iron deficiency. These may confirm that the sTfR/log ferritin or sTfR index can also provide a picture of iron reserves in the body in the CKDhemodialysis population who routinely receive Epo therapy.

The sTfR index is considered more accurate in assessing iron deficiency status in inflammatory conditions than conventional markers such as serum iron and ferritin. sTfR/log ferritin in inflammatory conditions such as a population with inflammatory bowel disease more accurately distinguishes anemia due to iron deficiency from anemia in chronic disease.(12) In conditions without inflammation, iron deficiency will also be described by decreased ferritin levels, but ferritin increases in inflammation. Thus, markers that are not affected by inflammation but still consider ferritin components such as sTfR/log ferritin are needed.

The sTfR/log ferritin was also found to detect iron deficiency anemia more accurately than other conventional iron status markers in 100 patients undergoing regular hemodialysis.(11) The study was conducted by giving intravenous iron supplementation of $100 \mathrm{mg} 3$ times/week for four weeks, followed by a dose of $100 \mathrm{mg}$ every two weeks for five months. Fifty-two patients were found to 
respond to intravenous iron therapy. Of the 52 respondent patients, only $14(27 \%)$ were found to meet the criteria for iron deficiency with routine iron examination markers (ferritin $<100$ and Tsat $<20 \%$ ). However, by examining iron status using sTfR / log Ferritin $>0.6$, it was found that about 63 percent of respondents met the iron deficiency criteria based on these markers. Further analysis found that sTfR/ $\log$ ferritin has a greater sensitivity (90\%) to detect iron deficiency than the combination of criteria ferritin $<100$ and Tsat $<20 \%$. The sTfR index has a critical role in diagnosing iron deficiency anemia, mainly if the serum ferritin level does not decrease.(13) It can be concluded from the results of this study and the synthesis of various relevant studies that sTfR/log ferritin has a negative and significant correlation with serum iron levels and transferrin saturation.

Table 3 presents data that serum iron levels are associated with inflammatory variables (HsCRP levels) as well as serum ferritin. However, this is not the case with transferrin saturation. Thus, in the CKD-hemodialysis population, generally in chronic inflammatory conditions, the iron status assessment was more consistent using transferrin saturation than serum iron levels. On the other hand, there was no significant relationship between InHsCRP and sTfR and ferritin. Besides being shown to be significantly associated with serum iron levels, sTfR/ $\log$ ferritin was even more consistent in inflammatory conditions because it was not associated with HsCRP levels.

Another study found that CRP levels $>10 \mathrm{mg} / \mathrm{L}$ are associated with chronic inflammation.(14) Serum CRP levels are closely related to the formation of proinflammatory cytokines such as interleukin (IL)-1 and tumor necrosis factor (TNF)- $\alpha$. One study found that the HsCRP level in the hemodialysis CKD phase was higher compared to non-hemodialysis CKD patients.(15) In the hemodialysis CKD phase, it was reported that inflammation, as indicated by increased CRP levels, was a predictor of cardiovascular disease. Reportedly, every $1 \mathrm{mg} / \mathrm{L}$ increase in plasma CRP will increase mortality by $3 \%$.(16) Previous study with 82 samples of people with regular HD obtained a positive correlation between CRP and ferritin, a correlation coefficient of $0.3(p=0.005)$.(17) A study of iron status, marked by ferritin and transferrin serum with inflammation also had been done in other study, which involved metabolic syndromes patients, in which ferritin corelated with HsCRP.(18)

It can be concluded that inflammation, as measured by HsCRP levels, was not associated with sTfR, sTfR/log ferritin but was significantly associated with serum iron and ferritin levels.

\section{Conclusion}

sTfR was associated with serum iron levels in chronic kidney disease patients undergoing hemodialysis, but not with neither serum transferrin nor ferritin saturation. Also, $\mathrm{sTfR} / \log$ ferritin was significantly associated with serum iron and transferrin saturation in patients with chronic kidney disease undergoing hemodialysis.

\section{Acknowledgements}

This study was supported by grants from the DIPA PNBP Universitas Udayana TA-2018 based on Assignment of Agreement Letter about Research Implementation Number:.61/UN 14.2.2/UPPM/2018 (May 11, 2018).

\section{Authors Contribution}

YK, GWM, IKS and IGRW designed the research, collect material and clinical data, analyzed the data, and writing the manuscript. WDL and SHW collect material and clinical data, and writing the manuscript. All authors have read and approved the final manuscript.

\section{References}

1. Bello AK, Levin A, Tonelli M, Okpechi IG, Feehally J, Harris D, et al. Global Kidney Health Atlas: A report by the International Society of Nephrology on the current state of organization and structures for kidney care across the globe. Brussels: International Society of Nephrology; 2017.

2. Urrechaga E, Borque L, Escanero J. Assessing iron status in CKD patients: new laboratory parameters. In: Monika Göőz, editor. Chronic Kidney Disease. London: InTech Open; 2012. doi: $10.5772 / 25645$.

3. Koulaouzidis A, Said E, Cottier R, Saeed AA. Soluble transferrin receptors and iron deficiency, a step beyond ferritin. A systematic review. J Gastrointestin Liver Dis. 2009; 18: 345-52.

4. Infusino I, Braga F, Dolci A, Panteghini M. Soluble transferrin receptor (sTfR), and sTfR/log ferritin index for the diagnosis of iron-deficiency anemia a meta-analysis. Am J Clin Pathol. 2012; 138: 642-9.

5. Beerenhout, C, Bekers O, Kooman JP, van der Sande FM, Leunissen KML. A comparison between the soluble transferrin receptor, transferrin saturation, and serum ferritin as markers of iron state in hemodialysis patients. Nephron. 2002; 92: 32-5.

6. Gaweda AE. Markers of iron status in chronic kidney disease. Hemodial Int. 2017; 21: S21-7.

7. Gupta D, Choudhary R, Sharma M, Saluja SGB. Role of soluble transferrin receptor and soluble transferrin receptor index in diagnosing iron deficiency anemia in patients with chronic kidney 
disease. Astrocyte. 2016; 3: 125-30.

8. Skikne BS, PunnonenK, Caldron PH, Bennett MT, Rehu M, Gasior $\mathrm{GH}$, et al. Improved differential diagnosis of anemia of chronic disease and iron deficiency anemia: A prospective multicenter evaluation of soluble transferrin receptor and the sTfR/log ferritin index. Am J Hematol. 2011; 86: 923-7.

9. Kurzawa T, Owczarek A, Strzelczyk J, Gołąbek K, Wiczkowski A. The content of reticulocyte hemoglobin and serum concentration of the soluble transferrin receptor for diagnostics of anemia in chronically hemodialyzed patients. Adv Clin Exp Med. 2016; 25: 425-31.

10. Bahrainwala J, Berns JS. Diagnosis of iron-deficiency anemia in chronic kidney disease. Semin Nephrol. 2016; 36: 94-8.

11. Chen Y, Hung S, Tarng D. Association between transferrin receptorferritin index and conventional measures of iron responsiveness in hemodialysis patients. Am J Kidney Dis. 2006; 47: 1036-44.

12. Oustamanolakis P, Koutroubakis IE, Messaritakis I. Soluble transferrin receptor-ferritin index in the evaluation of anemia in inflammatory bowel disease : a case-control study. Ann Gastroenterol. 2011; 24: 108-14.
13. Tijanić I, Vučić M, Golubović LM. The significance of soluble transferrin receptors in diagnosing iron deficiency anemia. Rev Rom Med. 2015; 23: 275-84.

14. Dhingra R, Gona P, Nam BH, D'Agostino RB, Wilson PWF, Benjamin EJ, et al. C-reactive protein, inflammatory conditions, and cardiovascular disease risk. Am J Med. 2007; 120: 1054-62.

15. Kadariswantiningsih IN, Thaha M, Nugroho CW, Hamidah B, Rasyid $\mathrm{H}$, Hakim ZE, et al. Could complete blood count parameters and non-fasting cholesterol profile describe inflammation and oxidative stress in chronic kidney disease?. Indones Biomed J. 2018; 10: 270-7.

16. Heidari B. C-reactive protein and other markers of inflammation in hemodialysis patients. Caspian Journal of Internal Medicine. 2013; 4: 611-6.

17. Kalantar-Zadeh K, Rodriguez RA, Humphreys MH. Association between serum ferritin and measures of inflammation, nutrition, and iron in hemodialysis patients. Nephrol Dial Transplant. 2004; 19: 141-9.

18. Nurfina W, Yusuf I, Arif M. Correlation of ferritin and transferrin serum with hsCRP and F2-isoprostane in metabolic syndrome. Indones Biomed J. 2010; 2: 131-6. 